\title{
Pairing symmetry and topological surface state in iron-chalcogenide superconductors
}

\author{
Lun-Hui Hu $\odot,{ }^{1}$ P. D. Johnson $\odot,{ }^{2}$ and Congjun Wu $\odot^{1}$ \\ ${ }^{1}$ Department of Physics, University of California, San Diego, California 92093, USA \\ ${ }^{2}$ Condensed Matter Physics and Materials Science Division, Brookhaven National Laboratory, Upton, New York 11973, USA
}

(Received 28 June 2019; accepted 11 February 2020; published 30 April 2020)

\begin{abstract}
The symmetries of superconducting gap functions remain an important issue in iron-based superconductivity. Motivated by the recent angle-resolved photoemission spectroscopic measurements of iron-chalcogenide superconductors, we investigate the influence of pairing symmetries on the topological surface state. If the surface Dirac cone becomes gapped in the superconducting phase, it implies magnetization induced from time-reversal symmetry-breaking pairing via spin-orbit coupling. Based on the crystalline symmetry constraints on the Ginzburg-Landau free energy, the gap function symmetries are among the possibilities of $A_{1 g(u)} \pm i A_{2 g(u)}$, $B_{1 g(u)} \pm i B_{2 g(u)}$, or $E_{g(u)} \pm i E_{g(u)}$. This time-reversal symmetry-breaking effect can exist in the normal state very close to $T_{c}$ with the relative phase between two gap functions locked at $\pm \frac{\pi}{2}$. The coupling between magnetization and superconducting gap functions is calculated based on a three-orbital model for the band structure of iron chalcogenides. This study provides the connection between the gap function symmetries and topological properties of the surface state.
\end{abstract}

DOI: 10.1103/PhysRevResearch.2.022021

The discovery of iron-based superconductors [1] has opened a new direction in the study of unconventional superconductivity [2-4]. Significant progress has subsequently been made in the search for new superconductors [5-19], and their pairing mechanisms have attracted considerable attention [20-24]. The parent compounds are metals with multiple Fermi surfaces around both $\Gamma$ and $M$ points. The possibility of the fully gapped extended $s_{ \pm}$-wave superconducting gap function is supported by various experimental evidence and theoretical calculations [25-33]. On the other hand, several theoretical studies suggest that $s_{ \pm}$and $d_{x^{2}-y^{2}}$ pairings are nearly degenerate in the iron-pnictide superconductors [26,34], leading to the possibility of a novel time-reversal (TR) symmetry-breaking pairing $s_{ \pm}+i d_{x^{2}-y^{2}}$ [35]. It was proposed that a resonance mode carrying the $B_{1 g}$ symmetry [35,36], which can be detected via Raman spectroscopy [37], exists if the $s_{ \pm}$and $d_{x^{2}-y^{2}}$ pairings are nearly degenerate. TR symmetry-breaking pairing also naturally arises in mixed singlet and triplet pairing states [38,39]. A chiral $d+i d$ pairing state is also found to spontaneously generate the gaps of the Haldane model [40].

Recently, the topological band structure of iron-based superconductors has aroused a great deal of attention. The $\mathrm{FeSe}_{1-x} \mathrm{Te}_{x}$ family with a wide range of composition $x$ is of particular interest [15,16,41-48].

Recent evidence shows that $\mathrm{FeSe}_{0.45} \mathrm{Te}_{0.55}$ is a strong topological insulator exhibiting a single Dirac cone on the (001) surface [49]. When the temperature is lowered below $T_{c}=$

Published by the American Physical Society under the terms of the Creative Commons Attribution 4.0 International license. Further distribution of this work must maintain attribution to the author(s) and the published article's title, journal citation, and DOI.
$14.5 \mathrm{~K}$, both bulk and the surface become superconducting [50,51]. The surface superconductivity is predicted to be topologically nontrivial $[45,46,52]$. In the same system, Majorana zero modes in vortex cores have been observed [53,54], exhibiting the signature of spin-selective Andreev reflection [55-57]. Similar evidence to vortex core Majorana modes is also observed in the $\left(\mathrm{Li}_{0.84} \mathrm{Fe}_{0.16}\right) \mathrm{OHFeSe}$ system [58].

However, the nontrivial topology of the surface superconductivity in $\mathrm{FeSe}_{0.5} \mathrm{Te}_{0.5}$ is mostly a property inherited from the normal state band structure in a similar way to the $\mathrm{Fu}-\mathrm{Kane}$ proposal of two-dimensional topological superconductivity via the proximity effect [59]. It does not directly reveal the symmetry properties of the superconducting gap functions. It would be highly desirable if the topological surface states could be used for phase-sensitive detections to unconventional pairing symmetries $[3,4]$. In contrast, in a very recent laser-based angle-resolved photoemission spectroscopy (APERS) experiment on $\mathrm{FeSe}_{0.3} \mathrm{Te}_{0.7}$ [60], the surface Dirac cone is observed to develop a gap as the system enters the superconducting state. The surface Dirac point is well below the Fermi energy. Thus the splitting cannot be the superconducting gap, but implies TR symmetry breaking in the spin channel, directly correlated with the superconducting transition. In earlier literature, both TR symmetry-breaking pairing of the types $s+i d$ [35,61-64] and $s+i s$ [65-68] have been proposed. However, in both cases magnetization only appears around impurities.

In the present work, we investigate how the topological surface states are affected by TR breaking gap functions, which in turn constrain the possible pairing symmetries. Note that the degenerate Dirac cone in the surface state is protected by TR symmetry in the normal state due to the nontrivial band structure topology. Nevertheless, if the superconducting state breaks TR symmetry, the degeneracy of the Dirac cone is no longer protected in the superconducting state. By employing 


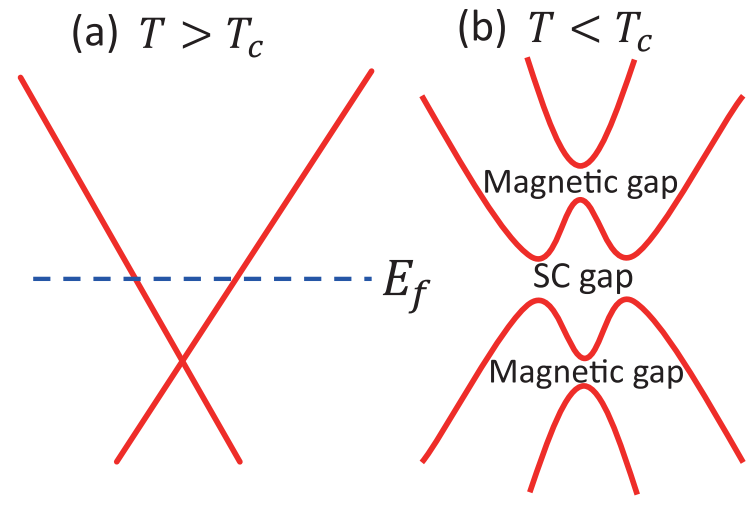

FIG. 1. Sketch of the surface spectra near the $\Gamma$ point. (a) In the normal state $\left(T>T_{c}\right)$, due to the nontrivial band structure topology, a surface Dirac cone develops inside the bulk band gap. (b) The surface state spectra in the superconducting state $\left(T<T_{c}\right)$. Two gaps appear: the superconducting gap at the chemical potential $\mu$ and the splitting of the Dirac cone due to the magnetic-order-breaking TR symmetry.

the Ginzburg-Landau formalism, we explore possible TR breaking gap functions which can induce magnetization via spin-orbit coupling to split the degeneracy at the surface Dirac point. Based on crystalline symmetry analysis, the superconductivity gap symmetries include the possibilities of $A_{1 g(u)} \pm$ $i A_{2 g(u)}, B_{1 g(u)} \pm i B_{2 g(u)}$, and $E_{g(u)} \pm i E_{g(u)}$. In the normal state sufficiently close to $T_{c}$, the relative phase between two gap functions can still be locked at $\pm \frac{\pi}{2}$ even though neither of them is long-range ordered. Calculations based on a threeorbital model are performed to derive the coupling between magnetization and superconducting gap functions. In doing so, our study bridges the topological properties of the surface state and the pairing symmetries of the superconducting gap functions.

We begin with a discussion of the splitting of the surface Dirac cone in the superconducting state. In the $\mathrm{FeSe}_{1-x} \mathrm{Te}_{x}$ materials, the degeneracy of the surface Dirac point is protected by the band topology if the normal state maintains TR symmetry. Consequently, a surface Dirac cone appears at the $\Gamma$ point, as shown in Fig. 1(a), described by an effective $k \cdot p$ Hamiltonian $H_{s f}=v\left(k_{x} \sigma_{y}-k_{y} \sigma_{x}\right)-\mu$, where $\sigma$ 's are Pauli matrices defined for the Kramers doublet at the $\Gamma$ point and $\mu$ is the chemical potential. A superconducting gap $\Delta$ by itself, i.e., $H_{\Gamma}=-\mu+\Delta w_{-}+\Delta^{*} w_{+}$where $w_{ \pm}=w_{x} \pm i w_{y}$ are Pauli matrices in the Nambu space of the particle-particle channel, does not lift the degeneracy. To split the degeneracy, a mass term breaking TR symmetry is necessary, i.e., $\Delta H_{\Gamma}=-m_{z} \sigma_{z}$, and the associated Bogoliubov spectra become $\pm m_{z}+\sqrt{|\Delta|^{2}+\mu^{2}}$ around the $\Gamma$ point. The sketch of the Bogoliubov dispersion is illustrated in Fig. 1(b) with both particle and hole branches. The mass term corresponds to a splitting between two eigenstates of $\sigma_{z}$. Since no magnetic field is applied, $m_{z}$ should arise from the Weiss field of a ferromagnetic ordering along the $z$ axis induced by superconductivity.

Now we examine how the ferromagnetic order $m_{z}$ can be induced in the superconducting state. Apparently, this requires the spontaneous breaking of TR symmetry. Indeed, within the Ginzburg-Landau (GL) formalism $[2,35,38,69,70]$, it has been shown that the mixing between two gap functions $\Delta_{1,2}$, which are TR invariant by themselves and possess different pairing symmetries, leads to the spontaneous TR symmetry breaking. The corresponding physical consequences were studied in the case of iron-based superconductors. $\Delta_{1,2}$ cannot form a symmetry invariant at the quadratic level, but they do at the quartic level via

$$
F_{4}=\beta\left|\Delta_{1}\right|^{2}\left|\Delta_{2}\right|^{2}+\beta^{\prime}\left(\Delta_{1}^{*, 2} \Delta_{2}^{2}+\text { c.c. }\right) .
$$

The $\beta^{\prime}$ term locks the relative phase between two gap functions, which equals $\beta^{\prime}\left|\Delta_{1}\right|^{2}\left|\Delta_{2}\right|^{2} \cos 2 \Delta \varphi$, where $\Delta \varphi=\varphi_{1}-$ $\varphi_{2}$ and $\varphi_{1,2}$ are the phases of two gap functions. When $\beta^{\prime}>0$, $\Delta \varphi$ is pinned at $\pm \frac{\pi}{2}$, giving rise to complex gap functions $\Delta_{1} \pm i \Delta_{2}$, which break TR symmetry spontaneously. This formalism also applies to the case in which $\Delta_{1,2}$ form a twodimensional (2D) irreducible representation. The complex gap functions $\Delta_{1} \pm i \Delta_{2}$ distribute more evenly over the Fermi surface than the real ones $\Delta_{1} \pm \Delta_{2}$, and hence, they are energetically more preferable at the mean-field level [38]. The corresponding Cooper pairs carry nonzero orbital moments, which could generate magnetic fields at boundaries as shown earlier $[69,70]$. However, these magnetic fields are typically of the order of 1 Gauss, for which the Zeeman energy is negligible. Instead, here we consider the spin magnetization $m_{z}$ coupling to $\Delta_{1,2}$ through a cubic term as

$$
\mathcal{F}_{M}=\alpha m_{z}^{2}+i \gamma m_{z}\left(\Delta_{1} \Delta_{2}^{*}-\Delta_{1}^{*} \Delta_{2}\right),
$$

This satisfies both the $U(1)$ and TR symmetry. $\alpha>0$ is assumed in Eq. (2), and hence there is no spontaneous magnetic ordering by itself; rather, the magnetization is induced, $m_{z}=\frac{\gamma}{\alpha}\left|\Delta_{1} \Delta_{2}\right| \sin \Delta \varphi$, i.e., by coupling to the TR breaking superconducting orders. The sign of $m_{z}$ is determined by the relative phase $\Delta \varphi$ between $\Delta_{1,2}$.

The free energy density $\mathcal{F}_{M}$ of Eq. (2) is further required to satisfy all crystalline symmetries. At elevated temperatures, the pristine FeSe and FeTe crystals are layered quasi-2D systems, whose Bravais lattices are primitive tetragonal. They exhibit a trilayer structure with each unit cell consisting of two $\mathrm{Fe}$ cations and two $\mathrm{Se}(\mathrm{Te})$ anions: A square lattice of $\mathrm{Fe}$ cations in the middle layer sandwiched between two layers of $\mathrm{Se}(\mathrm{Te})$ anions in a $\sqrt{2} \times \sqrt{2}$ structure. The $\mathrm{Se}(\mathrm{Te})$ lattices above and below the iron planes are offset by one Fe-Fe bond length, and their projections are at iron plaquette centers. The crystalline space group is the nonsymmorphic one $P 4 / \mathrm{nmm}$ $[16,43,50,51]$, which is reviewed in the Supplemental Material (SM) [71]. It can be decomposed into 16 cosets: 8 of them are denoted as $g_{i} T$ where $T$ is the translation group of the primitive tetragonal lattice and $g_{i}(i=1 \sim 8)$ span the point group $C_{4 v}$ centering at $\mathrm{Se}(\mathrm{Te})$ anions, and the other 8 cosets are $I g_{i} T$ by further applying the inversion $I$ with respect to the $\mathrm{Fe}-\mathrm{Fe}$ bond center. In the actual experimental systems of $\mathrm{FeSe}_{x} \mathrm{Te}_{1-x}$, the distribution of Se and Te breaks the $P 4 / \mathrm{nmm}$ symmetry; nevertheless, this effect is weak after averaging over random configurations and will be neglected below.

As shown in Fig. 2, the rotations with respect to $\mathrm{Se} / \mathrm{Te}$ and $\mathrm{Fe}$ are fourfold and twofold denoted as $C_{4}(z)$ and $C_{2}(z)$, respectively. The point group symmetry centering around the Fe cations is $D_{2 d}$. The vertical reflection planes along $x, y$ are denoted as $\sigma_{x}, \sigma_{y}$ and along the diagonal lines $x^{\prime}$ and $y^{\prime}$ are denoted as $\sigma_{x^{\prime}}$ and $\sigma_{y^{\prime}}$, respectively. 


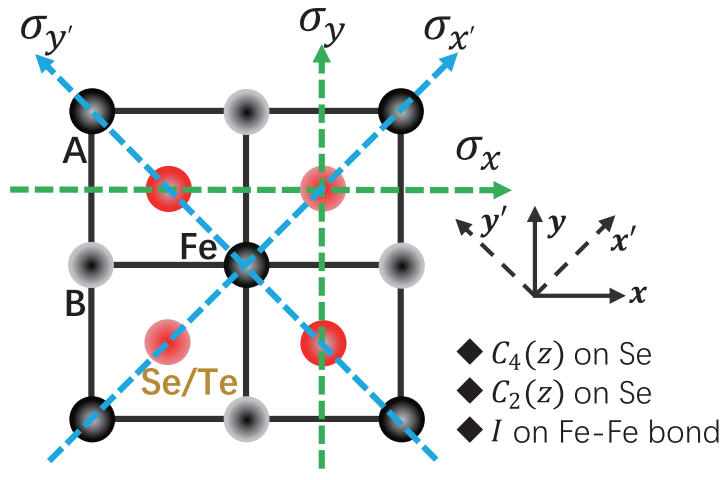

FIG. 2. Sketch of the lattice structure of a trilayer FeSeTe unit. The $z$ axis is perpendicular to the plane. The $P 4 / \mathrm{nmm}$ space group contains the $C_{4 v}$ point group symmetries centering at $\mathrm{Se}(\mathrm{Te})$ anions, the $D_{2 d}$ symmetries centering at Fe cations, and the inversion symmetries $I$ with respect to the Fe-Fe bond centers.

We consider the order parameter properties under the crystalline symmetries. In multiorbital systems, the superconducting gap function is expressed as

$$
\Delta=\sum_{\mathbf{k}} \tau_{i j} f^{l}(\mathbf{k}) M_{a b}^{l} \Delta_{b j, a i}(\mathbf{k}),
$$

where repeated indices mean summation; $a, b$ refer to the orbital band components and $i, j$ are the sublattice indices of two Fe cations in one unit cell; $\tau$ is a $2 \times 2$ matrix representing the sublattice channel; $f^{l}(\mathbf{k})$ is the angular form factor of momentum $\mathbf{k}$ and $M^{l}$ is the pairing matrix in the orbital channel; and $l$ is the index for multiple combinations between $f^{l}(\mathbf{k})$ and $M^{l}$. The pairing matrix is defined as $\Delta_{b j, a i}(\mathbf{k})=$ $\sum_{\mathbf{k}} i \sigma_{y, \alpha \beta}\left\langle\left|c_{\mathbf{k}, \alpha b j}^{\dagger} c_{-\mathbf{k}, \beta a i}^{\dagger}\right|\right\rangle$, where $i \sigma_{y}$ projects out the singlet pairing with Greek indices representing spin components, and $\langle||\rangle$ represents averaging over the thermal equilibrium state.

As required by Fermi statistics, for spin-singlet pairing, the product $\tau_{i j} f(\mathbf{k}) M_{a b}$ in Eq. (3) needs to be even under the combined operations of $\mathbf{k} \rightarrow-\mathbf{k}$ and the transposes of $M$ and $\tau . f(\mathbf{k})$ can be an even function taking the forms of 1 , $\cos k_{x} \pm \cos k_{y}, \cos k_{x} \cos k_{y}, \sin k_{x} \sin k_{y}, \cos \left(k_{x} \pm k_{y}\right)$, or an odd function among $\sin k_{x}, \sin k_{y}$, and $\sin \left(k_{x} \pm k_{y}\right)$. We choose the three $t_{2 g}$-orbital bases, $d_{x^{\prime} z}, d_{y^{\prime} z}$, and $d_{x y}$, where $d_{x^{\prime} z}\left(d_{y^{\prime} z}\right)$ extends along the diagonal $x^{\prime}\left(y^{\prime}\right)$ direction as depicted in the SM [71]. Hence, $M$ is a $3 \times 3$ Hermitian matrix which is expanded in terms of the Gell-Mann matrices $\lambda_{i}(i=1-8)$ under the basis in the sequence of $\left(d_{x^{\prime} z}, d_{y^{\prime} z}, d_{x y}\right)$, and the $3 \times 3$ identity matrix $\lambda_{0}$, whose expressions are presented in the SM [71].

The representation of a gap function under the crystalline symmetry group is determined by the symmetry properties of $f(\mathbf{k}), M$, and $\tau$ as analyzed and presented in the SM [71]. Their possible symmetries are denoted as $A_{1 g(u)}, A_{2 g(u)}$, $B_{1 g(u)}, B_{2 g(u)}$, and $E_{g(u)}$, respectively, where $g$ and $u$ represent even and odd parities, respectively. The $A, B$, and $E$ symbols represent the discrete angular momentum; loosely speaking, they are analogs to the $s-, d$-, and $p$-wave symmetries, respectively. $A_{1}$ and $A_{2}$ exhibit even and odd parities under vertical reflection planes; for example, the ferromagnetic order $m_{z}$ carries the $A_{2 g}$ symmetry. $B_{1}$ and $B_{2}$ are analogous to the $d_{x y}$ and $d_{x^{2}-y^{2}}$ symmetries, respectively, exhibiting opposite

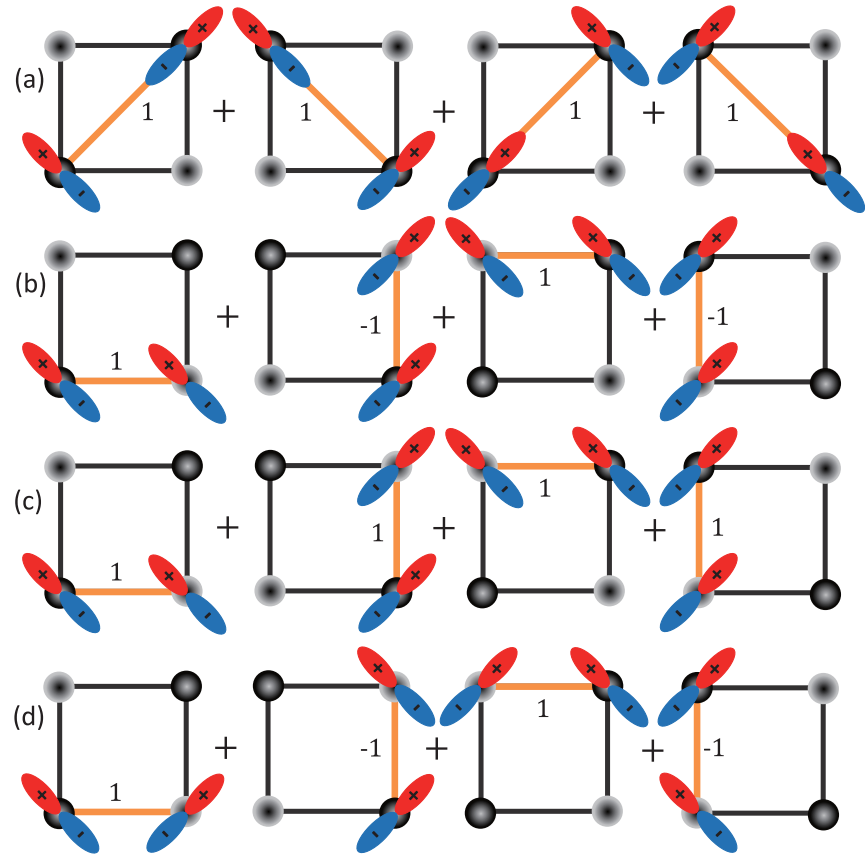

FIG. 3. The real space orbital configurations for the singlet pairing on a square plaquette of the Fe cations. The $B_{1 g}(a)$ and $B_{2 g}$ (b) pairings across the NNN and NN bonds, respectively, and their Fourier transforms give rise to the gap functions in Eq. (4). The $A_{1 g}$ (c) and $A_{2 g}(d)$ pairings across the NNN and NN bonds and their Fourier transforms give rise to gap functions in Eq. (5). Starting with the configuration of the first picture of each row, the subsequential configurations can be obtained by successively applying fourfold rotations.

parities under the $\sigma_{x(y)}$ and $\sigma_{x^{\prime}\left(y^{\prime}\right)}$ operations. Symmetries of singlet channel gap functions are classified accordingly: The next-nearest-neighbor (NNN) pairings are summarized in Table III and the nearest-neighbor (NN) pairings in Table IV in the SM [71]. Different combinations of $f(\mathbf{k}), M$, and $\tau$ often lead to the equivalent symmetries, and in general, the existence of one can induce others in the same symmetry class.

Many orbital-dependent gap functions have been proposed in the literature [72-75], and their importance has been analyzed in recent experiments [76,77]. Because of the multiorbital nature, the gap functions rigorously speaking cannot be intuitively represented by the partial-wave channels alone, i.e., the symmetry of the angular form factor $f(\mathbf{k})$. For example, consider the following two gap functions with even parity,

$$
\Delta_{1}: \cos k_{x} \cos k_{y} \tau_{0} \lambda_{1}, \quad \Delta_{2}: \cos k_{x} \cos k_{y} \tau_{0} \lambda_{3},
$$

which carry the $d_{x y^{-}}$and $d_{x^{2}-y^{2}}$-like symmetries, or, more precisely, $B_{1 g}$ and $B_{2 g}$ symmetries, respectively, although their angular form factors are $s$-wave like. They involve the intraand interorbital pairings between the $d_{x^{\prime} z}$ and $d_{y^{\prime} z}$ orbitals as shown in Fig. 3(a) and 3(b). Since $d_{x z} \rightarrow d_{y z}$ and $d_{y z} \rightarrow-d_{x z}$ under the $90^{\circ}$ rotation, $\lambda_{1,3}$ transform analogously in the $d$-wave way. By examining their reflection symmetries, they belong to the $B_{1 g}$ and $B_{2 g}$ symmetries. By similar analysis, the following gap functions exhibit the $A_{1 g}$ and $A_{2 g}$ symmetries, respectively, or, loosely speaking, the $s$-wave symmetry, in 
spite of their $d_{x^{2}-y^{2}}$ angular form factor:

$$
\Delta_{1}:\left(\cos k_{x}-\cos k_{y}\right) \tau_{1} \lambda_{3}, \quad \Delta_{2}:\left(\cos k_{x}-\cos k_{y}\right) \tau_{1} \lambda_{1} .
$$

Their orbital configurations are shown in Figs. 3(c) and 3(d). Furthermore, there can exist $p$-wave-like pairing symmetry or the $E_{g}$ symmetry in the singlet pairing channel,

$$
\Delta_{1}: \cos \left(k_{x}+k_{y}\right) \tau_{0} \lambda_{4}, \quad \Delta_{2}: \cos \left(k_{x}-k_{y}\right) \tau_{0} \lambda_{6},
$$

The former (latter) describes the pairing between the $d_{x^{\prime} z^{-}}$ orbital $\left(d_{y^{\prime} z}\right)$ with the $d_{x y}$ one.

The crystalline symmetries impose stringent constraints to the superconducting gap functions. According to Eq. (2), the direct product of the irreducible representations of $\Delta_{1}$ and $\Delta_{2}$ should contain that of $m_{z}$, i.e., $A_{2 g}$. This yields the following possibilities of pairing symmetries: $B_{1 g(u)} \pm i B_{2 g(u)}$, $A_{1 g(u)} \pm i A_{2 g(u)}$, and $E_{g(u)} \pm i E_{g(u)}$. Examples of the above pairing symmetries with even parity are provided in Eqs. (4), (5), and (6). They carry the same symmetry under rotation, and hence the experimental observables do not break the rotational symmetry. However, they mix different symmetries with respect to the vertical reflection plane, and such symmetries are also spontaneously breaking. For example, the magnetization $m_{z}$ is odd under such reflections.

An important issue is that spin-orbit coupling is necessary to break the SU(2) symmetry such that the ferromagnetic order $m_{z}$ and superconducting orders $\Delta_{1,2}$ can couple, since the former and latter are in the spin triplet and singlet channels, respectively. We employ a widely used threeband model for the topological band structure of $\mathrm{FeTe}_{1-x} \mathrm{Se}_{x}$ around the $\Gamma$ point, which consists of the $t_{2 g}$ orbitals $d_{x^{\prime} z}, d_{y^{\prime} z}$, and $d_{x y}[45,52]$. Neglecting the small dispersion along the $z$ axis, the three-band tight-binding Hamiltonian is expressed as [78] $H_{0}=\sum_{k} \psi^{\dagger}(k) \mathcal{H}_{0}(k) \psi(k)$, where $\psi=$ $\left[d_{x^{\prime} z, \uparrow}, d_{y^{\prime} z, \uparrow}, d_{x y, \uparrow}, d_{x^{\prime} z, \downarrow}, d_{y^{\prime} z, \downarrow}, d_{x y, \downarrow}\right]^{T}$ and the matrix kernel $\mathcal{H}_{0}(k)$ is given by

$$
\mathcal{H}_{0}=\mathcal{H}_{\mathrm{NNN}}+\mathcal{H}_{\mathrm{NN}}+\mathcal{H}_{\mathrm{soc}},
$$

where $\mathcal{H}_{\mathrm{NNN}}$ and $\mathcal{H}_{\mathrm{NN}}$ represent the NNN and NN hoppings, respectively, with detailed forms presented in the SM [71]. $\mathcal{H}_{\text {soc }}=\lambda_{\text {soc }} \tau_{0} \vec{L} \cdot \vec{\sigma}$ is the atomic spin-orbit coupling with $\lambda_{\text {soc }}$ being the coupling strength and $\vec{L}$ representing the onsite orbital angular momentum projected to the $t_{2 g}$ basis. Explicitly, $\vec{L}=\left[\left(\lambda_{5}-\lambda_{7}\right) / \sqrt{2},-\left(\lambda_{5}+\lambda_{7}\right) / \sqrt{2},-\lambda_{2}\right]$.

Based on the band structure, Eq. (7), the coupling coefficient $\gamma$ in Eq. (2) can be evaluated as

$$
\gamma=\frac{1}{\beta} \sum_{\mathbf{k}, \omega_{n}} f_{1}^{l_{1}}(\mathbf{k}) f_{2}^{l_{2}}(\mathbf{k}) \operatorname{Tr}\left[G_{h} M_{1}^{l_{1}} G_{e} \sigma_{z} G_{e} M_{2}^{l_{2}}\right],
$$

where $\beta=1 /\left(k_{B} T\right)$ is the inverse of temperature; $f_{i}^{l_{i}}$ and $M_{i}^{l_{i}}$ with $i=1,2$ are the angular form factors and orbital pairing matrix kernels of $\Delta_{1,2}$, respectively; $G_{e}\left(\mathbf{k}, i \omega_{n}\right)=$ $\left[i \omega_{n}-\mathcal{H}_{0}(\mathbf{k})\right]^{-1}$ is the Matsubara Green's function with $\omega_{n}=$ $(2 n+1) \pi / \beta$ and $G_{h}\left(\mathbf{k}, i \omega_{n}\right)=G_{e}^{*}\left(-\mathbf{k},-i \omega_{n}\right)$. As shown in the SM [71], the hole-like Fermi pockets around the $\Gamma$ point are mainly from the bonding states between two $\mathrm{Fe}$ sublattices; i.e., they are approximately eigenstates of $\tau_{1}$ with the eigenvalue of 1 . Hence, only tracing over the spin and orbital channels are needed, and only these gap functions characterized by $\tau_{0,1}$ are considered. Gap functions with $\tau_{2,3}$ are pairing between bonding and antibonding states between twosublattices, which will be neglected below.

Next, we present the examples of gap functions $\Delta_{1,2}$ leading to the spontaneous magnetization $m_{z}$. We begin with the cases of $B_{1 g(u)} \pm i B_{2 g(u)}$. For parity even, i.e., $B_{1 g} \pm i B_{2 g}$, we take $\Delta_{1,2}$ in the form of Eq. (4). As shown in the SM [71], after further reducing the band Hamiltonian Eq. (7) to a two-band model only based on $d_{x^{\prime}\left(y^{\prime}\right) z}$, Eq. (8) yields an analytic expression as

$$
\gamma \approx-\frac{7 \zeta(3)}{4 \pi^{3}} \frac{\lambda_{\mathrm{soc}} N_{0}}{\left(k_{B} T_{c}\right)^{2}}
$$

with $N_{0}$ being the density of states at the Fermi surface. Since $m_{z}$ is induced by the TR breaking pairings via spinorbit coupling, the coupling coefficient $\gamma$ is proportional to the spin-orbit coupling strength. A calculation based on the three-band Hamiltonian is also performed numerically, which yields consistent results (see the SM [71]). The parityodd case, i.e., $B_{1 u} \pm i B_{2 u}$, is also numerically checked to yield a nonzero $\gamma$, for example, with $\Delta_{1}: \tau_{0}\left[\sin \left(k_{x}+k_{y}\right) \lambda_{7}+\right.$ $\left.\sin \left(k_{x}-k_{y}\right) \lambda_{5}\right]$ and $\Delta_{2}: \tau_{0}\left[\sin \left(k_{x}+k_{y}\right) \lambda_{5}-\sin \left(k_{x}-k_{y}\right) \lambda_{7}\right]$. The above two cases also break the mirror symmetries of $\sigma_{x(y)}$ and $\sigma_{x^{\prime}\left(y^{\prime}\right)}$ spontaneously. They are topologically nontrivial, belonging to the $\mathrm{C}$ class supporting the chiral Majorana edge modes [79-82]. We have also studied both cases of $A_{1 g(u)} \pm i A_{2 g(u)}$, which also yield nonzero $\gamma$ 's as shown in the SM [71]. The nodal pairing gap functions presented in Eq. (5) are used for the even-parity case, and the nodeless pairing with $\Delta_{1}: \tau_{0}\left[\sin \left(k_{x}+k_{y}\right) \lambda_{5}+\sin \left(k_{x}-k_{y}\right) \lambda_{7}\right]$ and $\Delta_{2}: \tau_{0}\left[\sin \left(k_{x}+k_{y}\right) \lambda_{7}-\sin \left(k_{x}-k_{y}\right) \lambda_{5}\right]$ are used for the oddparity case. For the case of $E_{g(u)} \pm i E_{g(u)}$, we take the gap functions presented in Eq. (6) as an example. Since $E_{g(u)} \otimes$ $E_{g(u)}=A_{1 g} \oplus A_{2 g} \oplus B_{1 g} \oplus B_{2 g}$, it also yields a nonzero $\gamma$ as calculated in the SM [71], and consequently induces magnetization.

In strongly correlated superconductors, there exist strong superconducting phase fluctuations in the normal state close to $T_{c}$ [83]. In this case, the phases $\varphi_{1}$ and $\varphi_{2}$ of gap functions are disordered such that $\left\langle\Delta_{1,2}\right\rangle=0$, but $\left|\Delta_{1,2}\right|$ remains finite. The $\beta^{\prime}$ term in Eq. (1) can still pin the relative phase $\Delta \varphi= \pm \frac{\pi}{2}$. This transition breaks TR symmetry and its critical temperature $T^{\prime}>T_{c}$. We still expect a weaker but still finite $m_{z}$ in the temperature window between $T_{c}$ and $T^{\prime}$.

The bulk spin magnetization induced by TR-breaking pairing qualitatively explains the gap opening of the surface Dirac cone observed in the $\mathrm{FeSe}_{0.3} \mathrm{Te}_{0.7}$ superconductor [60]. We next briefly discuss the issue of the possible magnetic field generated by the bulk magnetization. Its upper bound is estimated to be 15 Gauss (see the SM [71]), which is still smaller than the lower critical magnetic field of the $\mathrm{FeSe}_{1-x} \mathrm{Te}_{x}$ superconductors $\left(H_{c 1} \sim 30\right.$ Gauss) $[84,85]$. The actual field due to spin magnetization should be much smaller than this bound; hence, it can be offset by the orbital magnetization such that the total magnetic field remains zero in the Meissner state $[86,87]$. This is consistent with the observation that neutron spectroscopy does not detect a bulk magnetic field [88]. A very weak but finite internal magnetic field around 0.15 Gauss is detected in the FeSe superconductor by the muon spin 
rotation measurement $(\mu \mathrm{SR})[89,90]$, which may arise from the imperfect screening due to impurities and domains.

Discussion and conclusion. In this article, we have studied how the TR symmetry-breaking superconducting states can gap out the topological surface modes in the ironchalcogenide superconductors. Spin-orbit coupling is necessary to break the $\mathrm{SU}(2)$ symmetry in the spin channel, such that it bridges the magnetic ordering and the TR breaking pairing states. Three classes of gap function symmetries can lead to such an effect based on group theory analyses: $A_{1 g(u)}+i A_{2 g(u)}, B_{1 g(u)}+i B_{2 g(u)}$, and $E_{g(u)}+i E_{g(u)}$. In strongly correlated superconductors, the superconducting phase fluctuations can also lock their relative phase at $\pm \frac{\pi}{2}$, breaking TR symmetry in the normal state.
This work builds connections between pairing symmetries in iron-based superconductors and their topological band structures. In particular, it is helpful for understanding the superconductivity-induced gap opening for the topological surface state recently discovered in the $\mathrm{FeTe}_{0.7} \mathrm{Se}_{0.3}$ [60].

Note added. Recently, we became aware of a work by Kawakami and Sato [91] which mainly discusses the topological odd-parity superconductivity in iron-based superconductors.

Acknowledgments. L.H., P.D.J., and C.W. acknowledge helpful discussions with J. Tranquada and G. D. Gu. L.H. and C.W. are supported by AFOSR FA9550-14-1-0168. P.D.J. is supported by the Office of Science, U.S. Department of Energy under Contract No. DE-SC001274.
[1] Y. Kamihara, T. Watanabe, M. Hirano, and H. Hosono, J. Am. Chem. Soc. 130, 3296 (2008).

[2] M. Sigrist, Rev. Mod. Phys. 63, 239 (1991).

[3] D. J. Van Harlingen, Rev. Mod. Phys. 67, 515 (1995).

[4] C. C. Tsuei and J. R. Kirtley, Rev. Mod. Phys. 72, 969 (2000).

[5] X. Chen, T. Wu, G. Wu, R. Liu, H. Chen, and D. Fang, Nature (London) 453, 761 (2008).

[6] G. F. Chen, Z. Li, D. Wu, G. Li, W. Z. Hu, J. Dong, P. Zheng, J. L. Luo, and N. L. Wang, Phys. Rev. Lett. 100, 247002 (2008).

[7] Z.-A. Ren, G.-C. Che, X.-L. Dong, J. Yang, W. Lu, W. Yi, X.-L. Shen, Z.-C. Li, L.-L. Sun, F. Zhou et al., EPL 83, 17002 (2008).

[8] M. Rotter, M. Tegel, and D. Johrendt, Phys. Rev. Lett. 101, 107006 (2008).

[9] K. Sasmal, B. Lv, B. Lorenz, A. M. Guloy, F. Chen, Y.-Y. Xue, and C.-W. Chu, Phys. Rev. Lett. 101, 107007 (2008).

[10] N. Ni, A. Thaler, J. Q. Yan, A. Kracher, E. Colombier, S. L. Bud'ko, P. C. Canfield, and S. T. Hannahs, Phys. Rev. B 82, 024519 (2010).

[11] Y. Nakai, T. Iye, S. Kitagawa, K. Ishida, H. Ikeda, S. Kasahara, H. Shishido, T. Shibauchi, Y. Matsuda, and T. Terashima, Phys. Rev. Lett. 105, 107003 (2010).

[12] X. Wang, Q. Liu, Y. Lv, W. Gao, L. Yang, R. Yu, F. Li, and C. Jin, Solid State Commun. 148, 538 (2008).

[13] S. V. Borisenko, V. B. Zabolotnyy, D. V. Evtushinsky, T. K. Kim, I. V. Morozov, A. N. Yaresko, A. A. Kordyuk, G. Behr, A. Vasiliev, R. Follath, and B. Buchner, Phys. Rev. Lett. 105, 067002 (2010).

[14] Y. Mizuguchi, F. Tomioka, S. Tsuda, T. Yamaguchi, and Y. Takano, Appl. Phys. Lett. 93, 152505 (2008).

[15] F.-C. Hsu, J.-Y. Luo, K.-W. Yeh, T.-K. Chen, T.-W. Huang, P. M. Wu, Y.-C. Lee, Y.-L. Huang, Y.-Y. Chu, D.-C. Yan et al., Proc. Natl. Acad. Sci. USA 105, 14262 (2008).

[16] M. H. Fang, H. M. Pham, B. Qian, T. J. Liu, E. K. Vehstedt, Y. Liu, L. Spinu, and Z. Q. Mao, Phys. Rev. B 78, 224503 (2008).

[17] G. F. Chen, Z. G. Chen, J. Dong, W. Z. Hu, G. Li, X. D. Zhang, P. Zheng, J. L. Luo, and N. L. Wang, Phys. Rev. B 79, 140509(R) (2009).

[18] J. Guo, S. Jin, G. Wang, S. Wang, K. Zhu, T. Zhou, M. He, and X. Chen, Phys. Rev. B 82, 180520(R) (2010).

[19] T. Qian, X.-P. Wang, W.-C. Jin, P. Zhang, P. Richard, G. Xu, X. Dai, Z. Fang, J.-G. Guo, X.-L. Chen, and H. Ding, Phys. Rev. Lett. 106, 187001 (2011).
[20] A. Chubukov, Annu. Rev. Condens. Matter Phys. 3, 57 (2012).

[21] F. Wang and D.-H. Lee, Science 332, 200 (2011).

[22] X. Chen, P. Dai, D. Feng, T. Xiang, and F.-C. Zhang, Natl. Sci. Rev. 1, 371 (2014).

[23] P. D. Johnson, G. Xu, and W.-G. Yin, Iron-Based Superconductivity, Springer Series in Materials Science (Springer, Berlin, 2015), Vol. 211.

[24] Q. Si, R. Yu, and E. Abrahams, Nat. Rev. Mater. 1, 16017 (2016).

[25] I. I. Mazin, D. J. Singh, M. D. Johannes, and M.-H. Du, Phys. Rev. Lett. 101, 057003 (2008).

[26] K. Kuroki, S. Onari, R. Arita, H. Usui, Y. Tanaka, H. Kontani, and H. Aoki, Phys. Rev. Lett. 101, 087004 (2008).

[27] D. Parker, O. V. Dolgov, M. M. Korshunov, A. A. Golubov, and I. I. Mazin, Phys. Rev. B 78, 134524 (2008).

[28] A. V. Chubukov, D. V. Efremov, and I. Eremin, Phys. Rev. B 78, 134512 (2008).

[29] Y. Bang, H.-Y. Choi, and H. Won, Phys. Rev. B 79, 054529 (2009).

[30] K. Seo, B. A. Bernevig, and J. Hu, Phys. Rev. Lett. 101, 206404 (2008).

[31] W.-Q. Chen, K.-Y. Yang, Y. Zhou, and F.-C. Zhang, Phys. Rev. Lett. 102, 047006 (2009).

[32] D. Wang, Y. Wan, and Q.-H. Wang, Phys. Rev. Lett. 102, 197004 (2009).

[33] L.-H. Hu, W.-Q. Chen, and F.-C. Zhang, Phys. Rev. B 91, 161108(R) (2015).

[34] S. Graser, T. Maier, P. Hirschfeld, and D. Scalapino, New J. Phys. 11, 025016 (2009).

[35] W.-C. Lee, S.-C. Zhang, and C. Wu, Phys. Rev. Lett. 102, 217002 (2009).

[36] D. J. Scalapino and T. P. Devereaux, Phys. Rev. B 80, 140512(R) (2009).

[37] T. Böhm, A. F. Kemper, B. Moritz, F. Kretzschmar, B. Muschler, H.-M. Eiter, R. Hackl, T. P. Devereaux, D. J. Scalapino, and H.-H. Wen, Phys. Rev. X 4, 041046 (2014).

[38] C. Wu and J. E. Hirsch, Phys. Rev. B 81, 020508(R) (2010).

[39] A. Hinojosa, R. M. Fernandes, and A. V. Chubukov, Phys. Rev. Lett. 113, 167001 (2014).

[40] P. M. R. Brydon, D. S. L. Abergel, D. F. Agterberg, and V. M. Yakovenko, Phys. Rev. X 9, 031025 (2019). 
[41] K.-W. Yeh, T.-W. Huang, Y.-1. Huang, T.-K. Chen, F.-C. Hsu, P. M. Wu, Y.-C. Lee, Y.-Y. Chu, C.-L. Chen, J.-Y. Luo et al., EPL 84, 37002 (2008).

[42] B. C. Sales, A. S. Sefat, M. A. McGuire, R. Y. Jin, D. Mandrus, and Y. Mozharivskyj, Phys. Rev. B 79, 094521 (2009).

[43] S. Li, Phys. Rev. B 79, 054503 (2009).

[44] L. C. C. Ambolode, K. Okazaki, M. Horio, H. Suzuki, L. Liu, S. Ideta, T. Yoshida, T. Mikami, T. Kakeshita, S. Uchida, K. Ono, H. Kumigashira, M. Hashimoto, D. H. Lu, Z. X. Shen, and A. Fujimori, Phys. Rev. B 92, 035104 (2015).

[45] Z. Wang, P. Zhang, G. Xu, L. Zeng, H. Miao, X. Xu, T. Qian, H. Weng, P. Richard, A. V. Fedorov, H. Ding, X. Dai, and Z. Fang, Phys. Rev. B 92, 115119 (2015).

[46] X. Wu, S. Qin, Y. Liang, H. Fan, and J. Hu, Phys. Rev. B 93, 115129 (2016).

[47] P. D. Johnson, H.-B. Yang, J. D. Rameau, G. D. Gu, Z.-H. Pan, T. Valla, M. Weinert, and A. V. Fedorov, Phys. Rev. Lett. 114, 167001 (2015).

[48] J. D. Rameau, N. Zaki, G. D. Gu, P. D. Johnson, and M. Weinert, Phys. Rev. B 99, 205117 (2019).

[49] P. Zhang, K. Yaji, T. Hashimoto, Y. Ota, T. Kondo, K. Okazaki, Z. Wang, J. Wen, G. Gu, H. Ding et al., Science 360, 182 (2018).

[50] H. Miao, P. Richard, Y. Tanaka, K. Nakayama, T. Qian, K. Umezawa, T. Sato, Y.-M. Xu, Y. Shi, N. Xu, X.-P. Wang, P. Zhang, H.-B. Yang, Z.-J. Xu, J.-S. Wen, G.-D. Gu, X. Dai, J.-P. Hu, T. Takahashi, and H. Ding, Phys. Rev. B 85, 094506 (2012).

[51] J. Yin, Z. Wu, J. Wang, Z. Ye, J. Gong, X. Hou, L. Shan, A. Li, X. Liang, X. Wu et al., Nat. Phys. 11, 543 (2015).

[52] G. Xu, B. Lian, P. Tang, X.-L. Qi, and S.-C. Zhang, Phys. Rev. Lett. 117, 047001 (2016).

[53] D. Wang, L. Kong, P. Fan, H. Chen, S. Zhu, W. Liu, L. Cao, Y. Sun, S. Du, J. Schneeloch et al., Science 362, 333 (2018).

[54] L. Kong and H. Ding, Natl. Sci. Rev. 6, 196 (2019).

[55] H.-H. Sun, K.-W. Zhang, L.-H. Hu, C. Li, G.-Y. Wang, H.-Y. Ma, Z.-A. Xu, C.-L. Gao, D.-D. Guan, Y.-Y. Li et al., Phys. Rev. Lett. 116, 257003 (2016).

[56] L.-H. Hu, C. Li, D.-H. Xu, Y. Zhou, and F.-C. Zhang, Phys. Rev. B 94, 224501 (2016).

[57] J. J. He, T. K. Ng, P. A. Lee, and K. T. Law, Phys. Rev. Lett. 112, 037001 (2014).

[58] Q. Liu, C. Chen, T. Zhang, R. Peng, Y.-J. Yan, C.-H.-P. Wen, X. Lou, Y.-L. Huang, J.-P. Tian, X.-L. Dong, G.-W. Wang, W.C. Bao, Q.-H. Wang, Z.-P. Yin, Z.-X. Zhao, and D.-L. Feng, Phys. Rev. X 8, 041056 (2018).

[59] L. Fu and C. L. Kane, Phys. Rev. Lett. 100, 096407 (2008).

[60] N. Zaki, G. Gu, A. M. Tsvelik, C. Wu, and P. D. Johnson, arXiv:1907.11602.

[61] C. Platt, R. Thomale, C. Honerkamp, S.-C. Zhang, and W. Hanke, Phys. Rev. B 85, 180502(R) (2012).

[62] M. Khodas and A. V. Chubukov, Phys. Rev. Lett. 108, 247003 (2012).

[63] R. M. Fernandes and A. J. Millis, Phys. Rev. Lett. 111, 127001 (2013).

[64] J. Kang, A. V. Chubukov, and R. M. Fernandes, Phys. Rev. B 98, 064508 (2018).

[65] V. Stanev and Z. Tešanović, Phys. Rev. B 81, 134522 (2010).

[66] M. Marciani, L. Fanfarillo, C. Castellani, and L. Benfatto, Phys. Rev. B 88, 214508 (2013).

[67] S. Maiti and A. V. Chubukov, Phys. Rev. B 87, 144511 (2013).
[68] F. Ahn, I. Eremin, J. Knolle, V. B. Zabolotnyy, S. V. Borisenko, B. Büchner, and A. V. Chubukov, Phys. Rev. B 89, 144513 (2014).

[69] G. Volovik and L. Gor'kov, Zh. Eksp. Teor. Fiz. 88, 1412 (1985), translated in English by J. G. Adashko, Sov. Phys. JETP 61, 843 (1985).

[70] M. Sigrist, in Lectures on the Physics of Highly Correlated Electron Systems IX: Ninth Training Course in the Physics of Correlated Electron Systems and High- $T_{c}$ Superconductors, edited by A. Avella and F. Mancini, AIP Conf. Proc. No. 789 (AIP, New York, 2005), pp. 165-243.

[71] See Supplementary Material http://link.aps.org/supplemental/ 10.1103/PhysRevResearch.2.022021 for the review of the $\mathrm{P} 4 / \mathrm{nmm}$ nonsymmorphic group, the classification of the gap function symmetry, the three-orbital tight-binding model and the reduced two-orbital model, the Green's functions, the effect of the surface Neel order, and an estimation of the internal magnetic field.

[72] E. M. Nica, R. Yu, and Q. Si, npj Quantum Mater. 2, 24 (2017).

[73] H. Hu, R. Yu, E. M. Nica, J.-X. Zhu, and Q. Si, Phys. Rev. B 98, 220503(R) (2018).

[74] T. Nakayama, T. Shishidou, and D. F. Agterberg, Phys. Rev. B 98, 214503 (2018).

[75] E. J. König and P. Coleman, Phys. Rev. B 99, 144522 (2019).

[76] P. O. Sprau, A. Kostin, A. Kreisel, A. E. Böhmer, V. Taufour, P. C. Canfield, S. Mukherjee, P. J. Hirschfeld, B. M. Andersen, and J. S. Davis, Science 357, 75 (2017).

[77] A. Kostin, P. Sprau, A. Kreisel, Y. Chong, A. Böhmer, P. Canfield, P. Hirschfeld, B. Andersen, and J. Davis, Nat. Mater. 17, 869 (2018).

[78] M. Daghofer, A. Nicholson, A. Moreo, and E. Dagotto, Phys. Rev. B 81, 014511 (2010).

[79] M. Z. Hasan and C. L. Kane, Rev. Mod. Phys. 82, 3045 (2010).

[80] X.-L. Qi and S.-C. Zhang, Rev. Mod. Phys. 83, 1057 (2011).

[81] A. Bansil, H. Lin, and T. Das, Rev. Mod. Phys. 88, 021004 (2016).

[82] C.-K. Chiu, J. C. Y. Teo, A. P. Schnyder, and S. Ryu, Rev. Mod. Phys. 88, 035005 (2016).

[83] V. J. Emery and S. A. Kivelson, Nature (London) 374, 434 (1995).

[84] C. Yadav and P. Paulose, New J. Phys. 11, 103046 (2009).

[85] M. Abdel-Hafiez, J. Ge, A. N. Vasiliev, D. A. Chareev, J. Van de Vondel, V. V. Moshchalkov, and A. V. Silhanek, Phys. Rev. B 88, 174512 (2013).

[86] D. V. Shopova and D. I. Uzunov, Phys. Rev. B 72, 024531 (2005).

[87] L. Chirolli, F. de Juan, and F. Guinea, Phys. Rev. B 95, 201110(R) (2017).

[88] Z. Xu, J. A. Schneeloch, M. Yi, Y. Zhao, M. Matsuda, D. M. Pajerowski, S. Chi, R. J. Birgeneau, G. Gu, J. M. Tranquada, and G. Xu, Phys. Rev. B 97, 214511 (2018).

[89] T. Watashige, Y. Tsutsumi, T. Hanaguri, Y. Kohsaka, S. Kasahara, A. Furusaki, M. Sigrist, C. Meingast, T. Wolf, H. v. Löhneysen, T. Shibauchi, and Y. Matsuda, Phys. Rev. X 5, 031022 (2015).

[90] K. Matsuura, T. Takenaka, Y. Sugimura, T. Shibauchi, K. Yamakawa, Q. Sheng, Z. Guguchia, Y. Uemura, Y. Cai, G. Luke et al., Bull. Am. Phys. Soc. (2019), http://meetings.aps. org/Meeting/MAR19/Session/E10.6.

[91] T. Kawakami and M. Sato, Phys. Rev. B 100, 094520 (2019). 